\title{
EIN SOLIDUS VON KONSTANTIN VII. UND ROMANOS II. AUS MAROSÚJVÁR/OCNA MUREŞ (RO)
}

\author{
PÉTER PROHÁSZKA
}

Archeologický ústav SAV, Akademická 2, 94921 Nitra, Slovakei

prohaszkapeter@freemail.hu

\begin{abstract}
A solidus of Constantine VII and Roman II from Marosújvár/Ocna Mures. The paper deals with a solidus of Constantine VII and Romanos II which was found in 1861 in Marosújvár (today Ocna Mureş, Romania). A drawing and a description of the finding circumstances was sent to Carl Torma, historian of antiquity by the engineer Franz Pošepny. From Transylvania only half a dozen Byzantine coins of the $9^{\text {th }}-10^{\text {th }}$ centuries are known. The majority of the Byzantine coins known from the Carpathian Basin belong to the reign of Constantine VII and Roman II, and were given to the Hungarians as a gift or subsidium. The author in his study gives an evaluation of it.
\end{abstract}

Keywords: Byzantine coins, $10^{\text {th }}$ century, Transylvania, archival records

\section{EINFÜHRUNG}

Die Verbreitung der landnahmezeitlichen byzantinischen Münzen im Karpatenbecken zeigt kein einheitliches Bild. In manchen Regionen des Karpatenbeckens kommen die Prägungen einzelner Kaiser ziemlich oft vor, aber es gibt auch solche Gebiete, wo trotz mehr als zwei Jahrhunderten Forschung kaum Münzen bekannt sind. ${ }^{1} \mathrm{Zu}$ diesen Gebieten gehört auch das in der südöstlichen Ecke des Karpatenbeckens liegende Siebenbürgen. Im Partium sowie im Gebiet des Banats kommen die byzantinischen numismatischen Denkmäler vor, aber aus Siebenbürgen sind kaum ein Dutzend byzantinische Münzen des 9. und 10. Jahrhunderts bekannt (Abb. 1). ${ }^{2}$ Die älteste Münze, eine gelochte Silbermünze von Basileios I. und Konstantin VI. (868-879), ist aus Csíkszépvíz ${ }^{3}$ (heute Frumoasa, jud. Harghita) bekannt. Die zeitlich nachfolgende - ein Solidus von Basileios II. und Konstantin VIII. (976-1025) - kam in der Umgebung von Gyulafehérvár (heute Alba Iulia, jud. Alba) ans Tageslicht. ${ }^{4}$ Ebenfalls aus der Zeit der ungarischen Staatsgründung stammen die anonymen Folles von Alsócsernáton (heute Cernatul de Jos, jud. Kovasna) ${ }^{5}$ und Medgyes (heute Medias, jud. Sibiu $)^{6}$ sowie das Stück aus dem Grab 46 von Vajdahunyad-Kincseshegy (heute Hunedoara, jud. Hunedoara). ${ }^{7}$ Außerdem ist ein Schatzfund aus Székelyudvarhely (heute Odorheiu Secuiesc, jud. Harghita) bekannt, welcher neben zwei Goldmünzen aus dem 7. Jahrhundert je einen Solidus der Kaiser Romanos I. und Christophoros, Konstantinos VII. und Romanos II., Basileios II. und Kostantinos VIII. (976-1025)

${ }^{1}$ Über die Verbreitung der byzantinischen Münzen s. Gedai 1969; KovÁCs 1989; ProhÁszKa 2012.

${ }^{2}$ Velter 2002, 73-74; vgl. Prohászka 2012; s. noch Gáll 2013. Bei dem von E. Gáll (GÁll 2013, 47) als Fund von Arad veröffentlichten Solidus des Konstantin VII. handelte es sich um eine Prägung von Romanos I. und Christophoros, deren Fundstelle im Komitat Temes lag: ProHÁszKa 2012, 70.
${ }^{3}$ ProhásZKa 2012, 69; vgl. Kovács 2011, 164 bzw. GÁLL 2013, 89. Beide führten die Münze als eine Prägung Leos VI. an, was aber falsch ist.

${ }^{4}$ PaVel 1977, 666-667; Gáll 2013, 200, Taf. 85.

${ }^{5}$ GÁll 2013, 38.

${ }^{6}$ GÁll 2013, 340.

${ }^{7}$ GÁll 2013, 514

Acta Archaeologica Academiae Scientiarum Hungaricae 69 (2018) 331-338 0001-5210 (C) 2018 Akadémiai Kiadó, Budapest 
sowie Nikephoros II. Phokas enthielt. ${ }^{8}$ Obwohl die archäologische Erforschung von Siebenbürgen schon in der zweiten Hälfte des 18. Jahrhunderts begann, ${ }^{9}$ sind kaum ein Dutzend byzantinische Münzen aus dem Gebiet bekannt. ${ }^{10}$ Eine Erklärung dafür wäre, dass das Hauptinteresse der Forschung bei den römischen Funden lag. In dieser

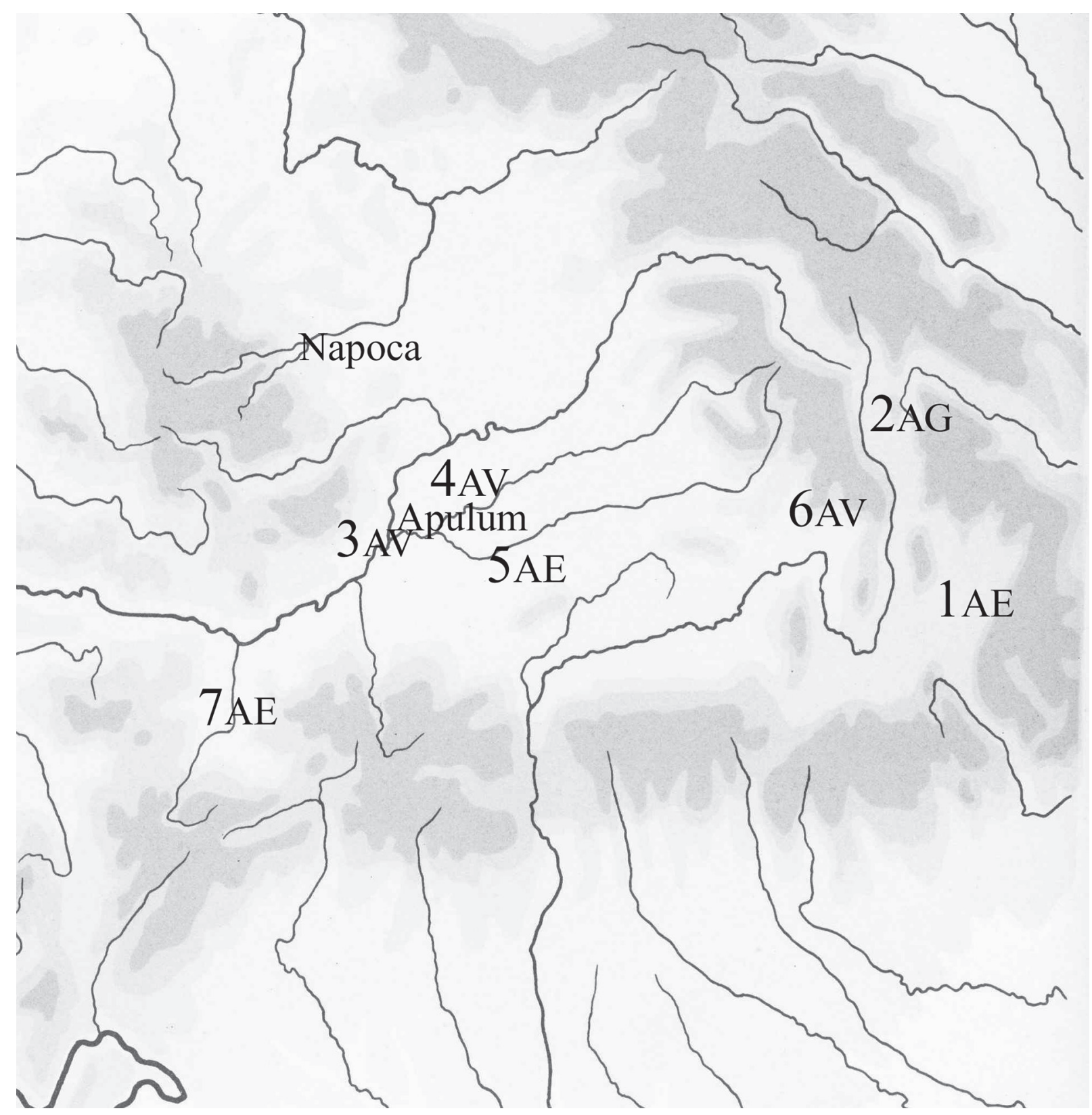

Abb. 1. Fundorte byzantinischer Münzen der Landnahmezeit und der ungarischen Staatsgründung in Siebenbürgen. 1: Alsócsernáton; 2: Csíkszépvíz; 3: Gyulafehérvár; 4: Marosújvár; 5: Medgyes; 6: Székelyudvarhely; 7: Vajdahunyad

${ }^{8}$ PROHÁSZKA 2012, 72.

${ }^{9}$ Vollmann 1983, 5-15. Hier müssen wir darauf hinweisen, dass hauptsächlich die Edelmetallmünzen eine große Beachtung durch die Forscher und Sammler erfuhren. Dagegen wurden die bronzenen bzw. kupfernen Prägungen kaum beschrieben oder gesammelt.
${ }^{10}$ Hier müssen wir betonen, dass zum so genannten „Fund von Vadas" keinerlei byzantinische Münzen gehörten. Nach dem Inventarbuch des Nationalmuseums wurden diese Münzen gewiss nicht in Siebenbürgen gefunden; siehe darüber ausführlich: PROHÁszKA 2007. 
Hinsicht ist der in Marosújvár gefundene und bisher unveröffentlichte Solidus von Konstantin VII. und Romanos II. besonders wichtig, über den sich Angaben in der Handschriftensammlung der Ungarischen Akademie der Wissenschaften befinden. ${ }^{11}$

\section{BESCHREIBUNG UND ZEICHNUNG VON FRANZ POŠEPNY ÜBER DEN SOLIDUS VON MAROSÚJVÁR}

Der Verfasser des Briefes war der Bergbauingenieur Franz/Frantisek Pošepny, der eine bedeutende Rolle in der geologischen Aufnahme des Siebenbürgischen Erzgebirges spielte. ${ }^{12}$ Zur archäologischen Forschung trug er hauptsächlich mit der Vermessung der Goldgruben zu Verespatak bzw. der Beschreibung der dort gefundenen römischen Werkzeuge bei. ${ }^{13}$ Pošepny ist am 30. März 1836 in der böhmischen Stadt Jilemnice geboren. Er studierte Ingenieurwissenschaften im Polytechnischen Institut zu Prag zwischen 1852 und $1857 .{ }^{14} 1859$ trat er in den staatlichen Dienst ein und wurde schon im nächsten Jahr nach Siebenbürgen, nach Nagybánya, geschickt, wo er mit der Aufnahme der Erzgruben beauftragt wurde. So gelangte er auch nach Verespatak (heute Rosia Montană). ${ }^{15}$ Er blieb bis 1869 in Siebenbürgen, dann kehrte er nach Böhmen zurück, wo er Professor der Bergbauakademie von Přibram wurde. Pošepny veröffentlichte zahlreiche Werke über Geologie bzw. Bergbaukunde. ${ }^{16}$ Wegen Verschlechterung seines gesundheitlichen Zustands musste er in Rente gehen und einige Jahre später starb Pošepny am 27. März 1895 in Wien. ${ }^{17}$ Bei seiner Tätigkeit in Siebenbürgen erwachte sein Interesse an verschiedenen Denkmälern der antiken Welt, weswegen er Kontakt mit Carl Torma (1829-1897), dem herausragendsten Epigraphiker und Archäologen dieser Epoche in Siebenbürgen, aufnahm. Pošepny sandte zahlreiche Briefe an Torma, in denen er über seine archäologischen Beobachtungen und Funde aus der Umgebung berichtete. ${ }^{18}$ In einem auf den 15. Mai 1867 datierten Brief berichtete der Ingenieur auch über eine byzantinische Goldmünze: ${ }^{19}$ „Gleichzeitig erhalt ich zur Ansicht eine jüngst in der Nähe von M. Ujvár gefundene Goldmünze, die sich gegenwärtig im Besitze Hn. Grüns Gippelpächter in M. Ujvár befindet. Ich füge eine c. 4 mal vergrösserte Zeichnung bei, und werden Dentrag der Form der Buchstaben Anschauungsfehler vorgekommen sein könnten, so füge ich Wachsabdrücke bei, welche freilich nicht als gelungen betrachten sind. Ich erlaube mir die Bitte, mir die Resultate der Bestimmung gütigst zukommen zu lassen.“ Auf der beigefügten Zeichnung steht folgender Satz (Abb. 2): ${ }^{20}$ „Goldmünze gefunden am Máros Durchstich bei Máros Ujvár.“ Die zwei beigelegten Wachsabdrücke gelangten wirklich nicht so gut. Zum Glück ist die Zeichnung von Pošepny genau geworden, obwohl es bei manchen Buchstaben der Umschrift kleinere Abweichungen gibt.

\section{DIE BESCHREIBUNG DER MÜNZE}

Nach der Zeichnung von Pošepny ist die Beschreibung der Münze wie folgt:

Avers: [+ IhS X]PS REX RE $\varsigma$ NANTIчmX - Brustbild von Christus frontal, um seinen Kopf ein Nimbus. Er trägt Pallium und Colobium. Die rechte Hand ist segnend erhoben, in der Linken hält er das Evangelium. Ein Fünftel der Münze ist ausgebrochen.

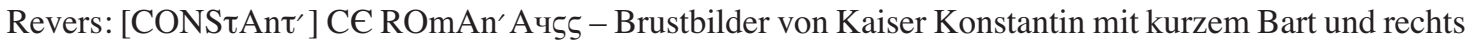
neben ihm dem bartlosen Romanos II. Beide tragen Kronen, zwischen den beiden ein Patriarchenkreuz mit langem Schaft. Der Kaiser trägt einen Loros und sein Sohn eine Chlamys.

Der Solidus gehört nach der Zeichnung zum Typ DOC 15, Münzstätte Konstantinopel (945-959) bzw. BMC 60.

\footnotetext{
${ }^{11}$ Schon früher kamen archivalische Angaben, Beschreibungen bzw. Fotos über byzantinische Münzen des 10.-11. Jahrhunderts zum Vorschein: ProhászKa 2004; ProhászKa 2012, 69-70.

${ }^{12}$ WINKLER 1871, 64-65.

${ }^{13}$ PoŠEPNY 1868; PošEPNy 1870.

${ }^{14} \mathrm{Zu}$ seinem Lebenslauf: ERNST 1895; LeBENSAFT 1983.

${ }^{15}$ ERNST 1895, VIII-IX.
}

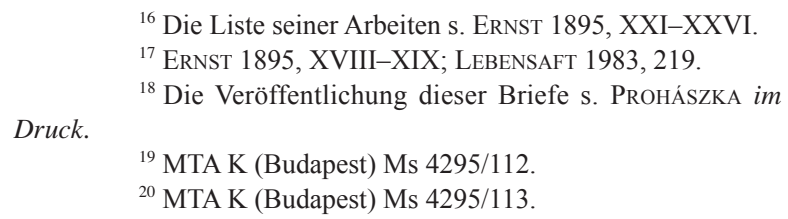




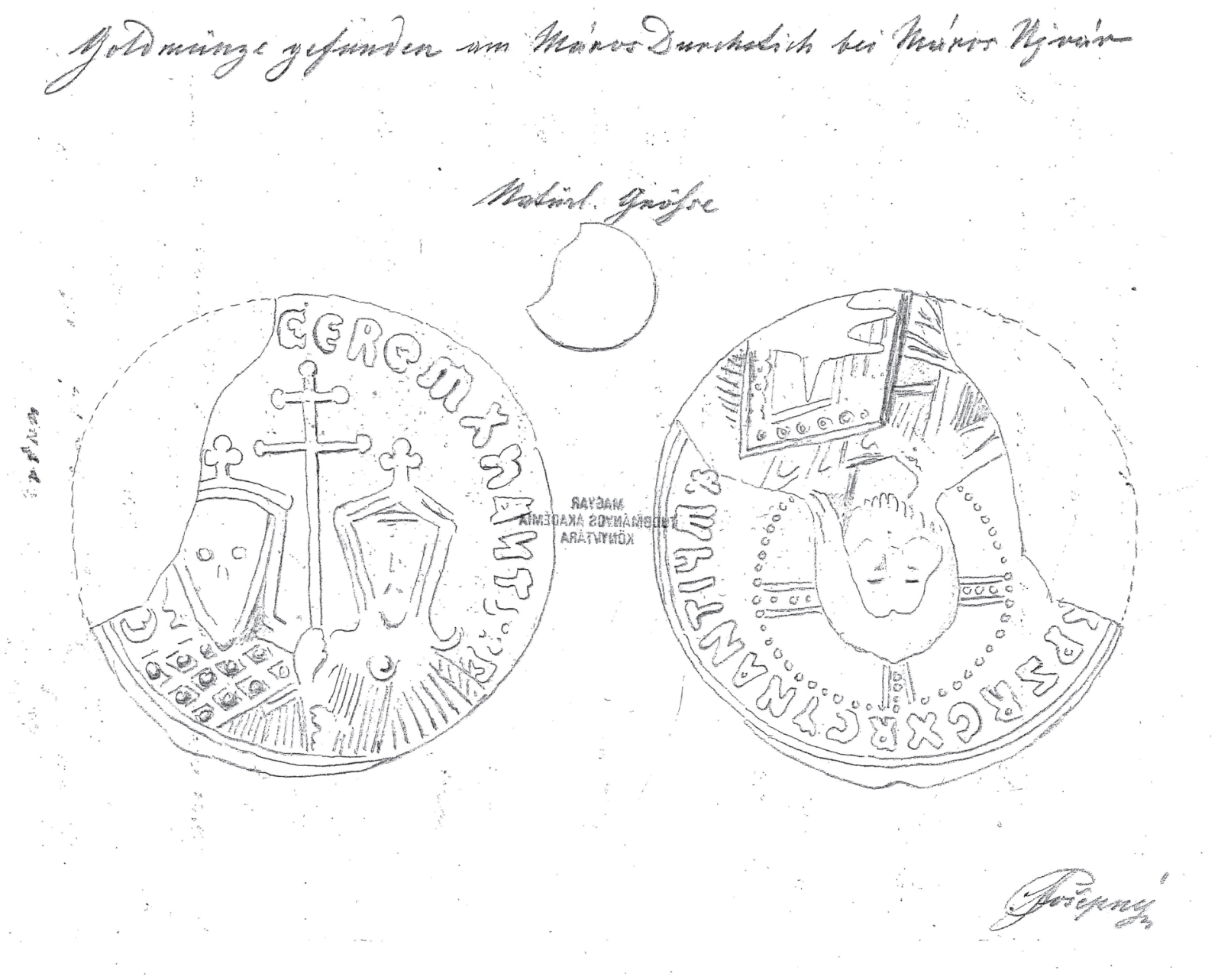

Abb. 2. Die Zeichnung von Franz/Frantisek Pošepny von dem Solidus von Marosújvár (MTA K)

AUSWERTUNG

Der Solidus von Marosújvár ist ein unikales Stück in Siebenbürgen. Bisher ist nur aus dem in der Epoche der Staatsgründung verborgenen Schatzfund von Székelyudvarhely ein Solidus der Kaiser Konstantin VII. und Romanos II. bekannt, ${ }^{21}$ obgleich diese zu den am häufigsten auftretenden byzantinischen Goldmünzprägungen im Karpatenbecken gehören. ${ }^{22}$ Die Münzen dieser Kaiser sind bisher aus 24 Fundorten im Karpatenbecken bekannt: Es sind meist Goldmünzen oder deren vergoldete Nachahmungen. ${ }^{23}$ Das häufige Auftreten der Prägungen von Konstantin VII. und Romanos II. kann man mit den aus den schriftlichen Quellen gut bekannten Ereignissen erklären.

Nach dem Streifzug gegen Thrakien im Jahre 934 griffen die Ungarn 943 wieder Byzanz an, was zu Verhandlungen mit Theophanes Patrikios führte. Byzanz schloss mit ihnen einen Friedensvertrag für fünf Jahre. ${ }^{24}$ Um 948 besuchten der ungarische Führer Bulcsu und nach ihm dann Gyula den Kaiserhof in Byzanz, wo sie getauft wurden und die Würde eines Patrikios erhielten, um dann mit viel Geld zurückzureisen. ${ }^{25}$ Über das Ereignis berichtet neben Iohannes Skylitzes ${ }^{26}$ auch Iohannes Zonaras. ${ }^{27}$

${ }^{21}$ ProhásZKa 2012, 72.

22 Siehe ProHÁsZKa 2012.

${ }^{23}$ ProhásZKa 2012, 80-81, vgl. die Tabelle unten.

${ }^{24}$ MoravcsiK 1953, 46; MoravcsiK 1984, 61-62, 85; MAKK 1996, 17
${ }^{25}$ GYÖRFFY 1977, 55-56; MAKK 1996, 17; BÓNA 2000, 63.

${ }^{26}$ MORAVCSIK 1984, 85-86.

${ }^{27}$ MoravCSIK 1984, 100. 
Mit diesen Ereignissen könnte die erwähnte Häufung der Solidi von Konstantinos VII. und Romanos II. in Verbindung stehen. Fast die Hälfte der Goldmünzen im Karpatenbecken stammt von diesen beiden Kaisern; sie kamen aus Gräbern (Vukovar-Lijeva bara Grab 2; žup. Vukovarsko-srijemska, Hr.) und aus Schatzfunden (Tokaj, Székelyudvarhely) zum Tageslicht. ${ }^{28}$ Diese Münzen sind entweder Zeugen der Jahresgelder nach 943 oder der Geschenke, welche den beiden Anführern überreicht wurden. ${ }^{29}$ Neben den Solidi sind fünf suberate Nachahmungen von Konstantin VII. und Romanos II. aus dem Karpatenbecken bekannt, welche auch als Grabbeigaben zum Vorschein gekommen sind. Sie könnten aus dem Byzantinischen Reich stammen und unter die ausgezahlten Solidi gemischt gewesen sein. Die kaiserliche Verwaltung probierte - wie früher bei den Awaren - mit Hilfe dieser Täuschung Gold einzusparen.

Von den Forschern der Landnahmezeit wird oft erwähnt, dass die byzantinischen Münzen vom Obertheißgebiet bis Orsova zum Vorschein gekommen seien, was durch die archäologischen Funde auch untermauert werde. ${ }^{30}$ Sie nahmen an, dass die Verbreitung das Herrschaftsgebiet des Fürsten Gyula zeige, der wegen seines Bündnisses Münzen, Schmuck und andere Geräte aus Byzanz erhalten habe. Obwohl eine Konzentration der Münzen in dem südlichen Teil der ungarischen Tiefebene wirklich beobachtbar ist, widersprechen die Münzen jener Vorstellung, dass sie ausschließlich in der Gegend der Flüsse Maros und Körös vorkämen. ${ }^{31}$ Wie die Verbreitung zeigt, treten die Münzen von Konstantin VII. und Romanos II. im ganzen Karpatenbecken auf (Abb. 3).

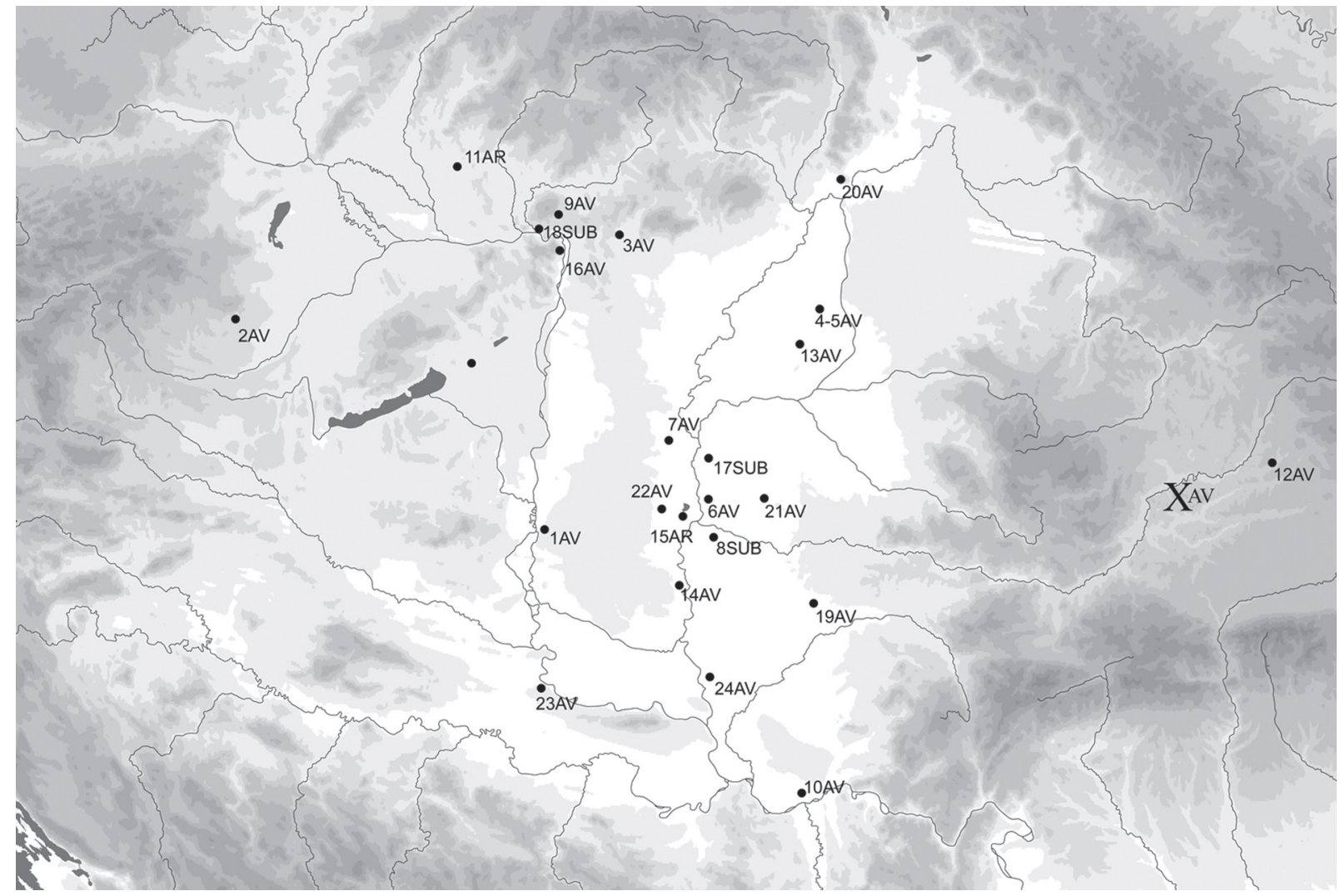

Abb. 3. Die Verbreitung der Münzen von Konstantin VII. und Romanos II. im Karpatenbecken

${ }^{28}$ KovÁcs $1989,137$.

${ }^{29}$ KRISTÓ 1980, 375; KovÁcs 1989, 143.
${ }^{30}$ BÓNA 2000, 64.

${ }^{31}$ Kristó 1980, 375; vgl. KovÁcs 1989, 143.

Acta Archaeologica Academiae Scientiarum Hungaricae 69, 2018 


\begin{tabular}{|c|c|c|c|}
\hline Nr. & Fundort & Bestimmung & Literatur \\
\hline 1 & Baja (Kom. Bács-Kiskun/H) & Solidus & PROHÁSZKA 2003-2004, 149. \\
\hline 2 & Burg [Sámfalva] (KG Burg, Burgenland/A) & Solidus BMC 60 (945-959) & Kaus 2000, 11-12. \\
\hline 3 & Gyöngyöspata (Kom. Heves/H) & Solidus BMC 60 (945-959) & KovÁCs 1989, 84, Nr. 906. \\
\hline 4 & Hajdúszoboszló (Kom. Hajdú-Bihar/H) & Solidus BMC 60 (945-959) & $\begin{array}{l}\text { KovÁCS 1989, 31, Nr. 99; } \\
\text { PROHÁSZKA 2003-2004, } 150 .\end{array}$ \\
\hline 5 & $\begin{array}{l}\text { Hajdúszoboszló-Árkoshalom Grab } 109 \\
\text { (Kom. Hajdú-Bihar/H) }\end{array}$ & Solidus BMC 60 (945-959) & M. NEPPER 2002, 74. \\
\hline 6 & Hódmezővásárhely-Kopáncs Grab 13 (Kom. Békés/H) & Suberatus nach Typ BMC $66 \bullet$ & KovÁCs 1989, 32, Nr. 113. \\
\hline 7 & Kiskunfélegyháza (Kom. Bács-Kiskun/H) & Solidus BMC 60 (945-959) & KovÁcs 1989, 176, Nr. 1122. \\
\hline 8 & Kiszombor B Grab 342 (Kom. Csongrád/H) & Suberatus nach Typ BMC 60-66• & KovÁcs 1989, 41, Nr. 198. \\
\hline 9 & Kóspallag-Szent István utca (Kom. Pest/H) & Solidus BMC 64 (945-959) & KovÁCs 1989, 90, Nr. 1041. \\
\hline 10 & Kovin [Kevevára] (Južnobanatski obl./SRB) & Solidus BMC 62 (945-959) & PROHÁSZKA 2012, 70. \\
\hline 11 & Nitra-Šindolka F Grab 11 (okr. Nitra/SK) & $\begin{array}{l}\text { Miliaresion, ein halbes Stück BMC } \\
?(945-959)\end{array}$ & HUNKA 2009, 399. \\
\hline $\mathrm{X}$ & Ocna Mureş [Marosújvár] (jud. Alba/RO) & Solidus BMC 60 (945-959) & S. im Beitrag. \\
\hline 12 & $\begin{array}{l}\text { Odorheiu Secuiesc [Székelyudvarhely] (jud. Harghita/RO) } \\
\text { Schatzfund }\end{array}$ & Solidus BMC 62 (945-959) & PROHÁSZKA 2012, 72-73. \\
\hline 13 & Sárrétudvar-Hízóföld Grab 112 (Kom. Hajdú-Bihar/H) & $\begin{array}{l}\text { Miliaresion BMC } 67 \text { (945-959) } \\
\text { Miliaresion BMC } 67 \text { (945-959) }\end{array}$ & M. NEPPER 2002, 316. \\
\hline 14 & Senta-Homoki szőlők [Zenta] (Severnobanatski obl./SRB) & Solidus & KovÁCs 1989, 77, Nr. 450. \\
\hline 15 & Szeged-Csongrádi út Grab 1 (Kom. Csongrád/H) & Miliaresion BMC 67-69 (948-959) & KovÁCs 1989, 61, Nr. 322. \\
\hline 16 & Szentendre (Kom. Pest/H) & Solidus BMC 60 (945-959) & PROHÁSZKA 2012, 68; KovÁCs 1989, 84, Nr. 901. \\
\hline 17 & Szentes-Borbásföld Grab 1 (Kom. Csongrád/H) & Suberatus nach Typ BMC $66 \bullet$ & KovÁCs 1989, 62, Nr. 332. \\
\hline 18 & Szob-Kiserdő Grab 60 (Kom. Pest/H) & Suberatus nach Typ BMC $60 \bullet$ & KovÁCs 1989, 67, Nr. 366. \\
\hline 19 & Timişoara [Temesvár] (jud. Timiş/RO) & Solidus BMC 60 (945-959) & KovÁcs 1989, 84, Nr. 903. \\
\hline 20 & „Tokaj“ (Kom. Borsod-Abaúj-Zemplén/H) Schatzfund & 10 Solidus BMC 60 (945-959) & KovÁCs 1989, 72, Nr. 403-412. \\
\hline 21 & Tótkomlós (Kom. Békés/H) & Solidus & KovÁCs 1989, 73, Nr. 413. \\
\hline 22 & Üllés (Kom. Csongrád/H) & Solidus BMC 60-66 (945-959) & KovÁCs 1989, 91, Nr. 1045. \\
\hline 23 & Vukovar-Lijeva bara Grab 2 (Vukovarsko-srijemska obl./HR) & Solidus BMC 60-64 (945-959) & KovÁCs 1989, 77, Nr. 445. \\
\hline 24 & Zrenjanin [Nagybecskerek] (Srednjobanatski obl./SRB) & Solidus BMC 64 (945-959) & KovÁCs 1989, 84, Nr. 907. \\
\hline
\end{tabular}

Die Verbreitung der Münzen von Konstantin VII. und Romanos II. im Karpatenbecken

Im Jahre 958 stellte Byzanz die Geldzahlungen ein ${ }^{32}$ was 959 zu einem ungarischen Kriegszug führte, welcher aber in einer Niederlage endete. ${ }^{33}$ Trotz der Niederlage erscheinen die Münzen des Nikephoros II. Phokas noch im Karpatenbecken. Neben den Solidi aus Orsova und aus den Schätzen zu Gaj und Székelyudvarhely kommen ein Subaeratus und ein Miliaresion vor. ${ }^{34}$ Den Quellen nach haben die Ungarn einen Raubzug durchgeführt, der durch das siegreiche Eingreifen der byzantinischen Truppen endete. Der Kaiser ließ danach ungarische Kriegsgefangene in seine Leibwache eintreten. Das Erscheinen der Solidi könnte auch auf erneute Verbindungen mit der byzantinischen Regierung hindeuten. Der Solidus von Konstantin VII. und Romanos II. aus Marosújvár gelangte höchstwahrscheinlich auf eine der oben dargelegten Wegen ins Karpatenbecken.

Das Fundmaterial des 10. und 11. Jahrhunderts in Siebenbürgen zeigt manche eigenartigen Erscheinungen, welche oft zu einander widersprechenden Folgerungen sowohl in der archäologischen als auch in der numismatischen Forschung führten. Mit dem Münzverkehr des 10. Jahrhunderts in Siebenbürgen beschäftigte sich zuletzt László Révész in seiner Abhandlung. ${ }^{35}$ Seiner Meinung nach könnte der Mangel an byzantinischen Münzen wichtige Hinweise bringen, dass die dort angesiedelten Gruppen entweder nicht an den Streifzügen nach Süden zwischen 955 und 971 teilnahmen oder in dieser Periode noch nicht dort ansässig waren. ${ }^{36}$ Wenn er aber der Verbreitung der byzantinischen Münzen in der erwähnten Epoche Beachtung geschenkt hätte, dann hätte er gewiss gemerkt, dass

\footnotetext{
${ }^{32}$ MAKK 1996, 22.

${ }^{33}$ MoravcsiK 1953, 48; Moravcsik 1984, 69; MaKK 1996,23
}

\footnotetext{
${ }^{34}$ Kovács 1989, 70; vgl. eine weitere Goldmünze des Nikephoros II. Phokas, die bei der Ausgrabung auf dem Sibrik Hügel in Visegrád zum Vorschein gekommen ist: BoRUZS-MERVA-SZABÓ 2018, 8.

${ }^{35}$ RÉVÉsz 2013, 615.

${ }^{36}$ RÉVÉSZ 2013, 615.
} 
aus dem dritten Viertel des 10. Jahrhunderts aus dem ganzen Karpatenbecken besonders wenig byzantinische Münzen bekannt sind. ${ }^{37}$ Ebenfalls stellte er in Frage, ob das Erscheinen der Münzen von Alsócsernáton, Csíkszépvíz und Medgyes mit der Ansiedlung der Ungarn in Verbindung stehe. ${ }^{38}$ Zum Glück hat er sich nicht jene Vorstellung von Erwin Gáll zu Eigen gemacht, wonach die kupfernen anonymen Folles die Denkmäler der byzantinischen Rückeroberung gewesen wären. ${ }^{39}$ Jedoch gab auch er keine Erklärung dafür, auf welche Art diese Münzen nach Siebenbürgen gelangt sind, wenn sie nicht durch die Ungarn hierher gebracht wurden.

In Hinsicht des Münzverkehrs vom Karpatenbecken gelangte der Solidus aus Marosújvár mit den sich entlang des Flusses Maros im Laufe des 10. Jahrhunderts schrittweise ansiedelnden Ungarn nach Siebenbürgen. Aber gewiss nicht durch eine byzantinische Botschaft oder durch slawische bzw. bulgarische Gruppen. ${ }^{40}$ Obwohl uns aus Marosújvár keine Angaben über Funde und Gräber aus dem 10. Jahrhundert zur Verfügung stehen ${ }^{41}{ }^{4}$ rscheinen in der Nähe des Ortes, am mittleren Teil des Marostals, im zweiten Drittel des 10. Jahrhunderts nacheinander mittelgroße bzw. große ungarische Gräberfelder - so zum Beispiel Gyulafehérvár-Brânduşei Straße, Gyulafehérvár császár forrása (Quelle des Kaisers) und Rettungsstation, Marosgombás, Magyarlapád bzw. Szászváros. ${ }^{42}$ Höchstwahrscheinlich könnte im Besitz einer zu diesen Gruppen gehörenden Person jener byzantinische Solidus von Konstantin VII. und Romanos II. gewesen sein, der 1861 in Marosújvár zum Vorschein gekommen ist.

\section{DANKSAGUNG}

Die Fertigstellung dieses Beitrags ermöglichte das SASPRO Programm der Slowakischen Akademie der Wissenschaften und der Europäischen Union (0104/01/03). Ich möchte Prof. Dr. Hubert Emmerig (Wien) für die muttersprachliche Korrektur des vorliegenden Textes danken.

\section{LITERATUR}

$\mathrm{BMC}$

BÓNA 2000

BORUZS-MERVA-SZABÓ 2018

DOC

ERNST 1895

GÁLl 2013

GEDAI 1969

GYÖRFFY 1977

HUNKA 2009
= W. W. Wroth: Catalogue of the Imperial Byzantine Coins in the British Museum. Vol. 2. London 1908.

= I. BóNA: A magyarok és Európa a 9-10. században [Die Ungarn und Europa im 9.-10. Jahrhundert]. Budapest 2000.

= K. BORUZS-Sz. MERVA-B. S. SZABÓ: Előzetes jelentés a Visegrád, Sibrik-dombon végzett tervásatásról [Vorbericht über die Plangrabung in Visegrád, Sibrik-domb]. Altum Castrum online. Visegrád 2018, 1-11.

$=$ P. Grierson-A. R. Bellinger: Catalogue of the Byzantine Coins in the Dumbarton Oaks Collection and in the Whittemore Collection. III.: Leo III to Nicephorus III (717-1081). Washington 1993.

= C. v. ERNST: Bergrath und Professor der Bergakademie Přibran. Archiv für practische Geologie 2 (1895) V-XX.

= E. GÁlL: Az Erdélyi-medence, a Partium és a Bánság 10-11. századi temetői, szórvány- és kincsleletei $\left(10^{\text {th }}\right.$ and $11^{\text {th }}$ century burial sites, stray finds and treasures in the Transylvanian basin, the Partium and the Banat). Magyarország honfoglalás kori és Árpád-kori sírleletei 6. Szeged 2013.

= I. GEDAI: Fremde Münzen im Karpatenbecken aus den 11.-13. Jahrhunderten. ActaArchHung 21 (1969) 105-148.

= Gy. GYÖRFFY: István király és müve [König Stephan und sein Werk]. Budapest 1977.

$=\mathrm{J}$. HunKA: Finds of Byzantine coins from the $5^{\text {th }}-10^{\text {th }}$ century from the northern part of the Carpathian Basin. In: Byzantine Coins in Central Europe between the $5^{\text {th }}$ and $10^{\text {th }}$ Century. Proceedings from the conference organised by Polish Academy of Arts and Sciences and Institute of Archaeology University of Rzeszów under patronae of Union Académique International (Programme No. 57
${ }^{37}$ ProhÁsZKA 2012, 81-82 und Abb. 8.

${ }^{38}$ RÉVÉSZ 2013, 615.

${ }^{39}$ Gáll 2013, 557, wo der Autor bei einigen im Szekler Nationalmuseum verwahrten anonymen Folles ohne Fundortangabe jene Möglichkeit aufwarf, dass sie auch mit der byzantinischen Reconquista/Rückeroberung in Verbindung stehen könnten. Diese ist in der Hinsicht besonders interessant, dass dieses Gebiet nie zum Byzan- tinischen Reich gehörte. Zum Glück war László Révész sowohl Opponent der Promotionsarbeit von Erwin Gáll als auch Lektor des aus dieser Arbeit entstandenen Buches.

${ }^{40}$ RÉVÉSZ 2013, 610.

${ }^{41}$ GÁll 2013.

${ }^{42}$ Gáll 2013, 817; RÉVÉsz 2013, 610, 614. 
KaUs 2000

KovÁCs 1989

KovÁcs 2011

KRISTÓ 1980

LEBENSAFT 1983

MAKK 1996

MoRAVCSIK 1953

MORAVCSIK 1984

M. NEPPER 2002

PAVEl 1977

POŠEPNY 1868

POŠEPNY 1870

PROHÁSZKA 2004

ProhÁSZKA 2007

PROHÁSZKA 2012

PROHÁSZKA 2013

ProhászKa im Druck

RÉVÉSZ 2013

VELTER 2002

VOLLMANN 1983

WINKLER 1871
Moravia Magna) Kraków, 23-26 IV 2007. Ed.: M. Wołoszyn. Moravia Magna, Seria Pologna 3. Kraków 2009, 395-401.

= K. KAus: Burg/Pinkaóvár-Tobolyvár, Kőbánya. In: G. Kiss: Vas megye 10-12. századi sír- és kincsleletei. Magyarország honfoglalás kori és kora Árpád-kori sírleletei 2. Szombathely 2000, 11-12.

= L. KovÁcs: Münzen aus der ungarischen Landnahmezeit. Archäologische Untersuchung der arabischen, byzantinischen, westeuropäischen und römischen Münzen aus dem Karpatenbecken des 10. Jahrhunderts. FontArchHung. Budapest 1989.

= L. KovÁcs: A magyar kalandozások zsákmányáról [Über die Beute der ungarischen Streifzüge]. Budapest 2011.

= GY. KRISTó: Levédi törzsszövetségétől Szent István államáig [Vom Stammesverband von Levedi bis zum Staat von Stephan dem Heiligen]. Budapest 1980.

= E. LeBENSAFT: Pošepny Franz. In: Österreichisches Biographisches Lexikon 1815-1950. ÖBL 8. Wien 1983, 219.

= F. MAKK: Magyar külpolitika (896-1196) [Ungarische Außenpolitik (896-1196)]. Szeged 1996.

= Gy. MoravcsiK: Bizánc és a magyarság (Byzantium and the Magyars). Budapest 1953.

= Gy. MoravcsiK: Az Árpád-kori magyar történelem bizánci forrásai (Fontes Byzantini historiae Hungaricae aevo ducum et regum ex stirpe Árpád descendentium). Budapest 1984.

= I. M. NEPPER: Hajdú-Bihar megye 10-11. századi sírleletei [Grabfunde im Komitat Hajdú-Bihar aus dem 10.-11. Jahrhundert]. Magyarország honfoglalás kori és kora Árpád-kori sírleletei 3. BudapestDebrecen 2002.

= V. PAVEL: Monede de aur imperiale şi bizantine în colecţiile muzeului din Alba Iulia (Monnaies Romaines et Byzantines en or de la Collection du Musée d'Alba Iulia). Apulum 15 (1977) 663-670.

= F. PoŠEPNY: Eine Bergmaschine aus dem 2. Jahrhundert und einige gleichzeitig gefundene Gegenstände. Österreichische Zeitschrift für Berg- und Hüttenwesen 16 (1868) 153-168.

= F. POŠEPNY: Vorlage der geologisch-montanistischen Generalkarte des Bergbau-Reviers vom Verespatak in Siebenbürgen. Verhandlungen der k. k. geologischen Reichanstalt Nr. 5.-7. Wien 1870.

= P. PROHÁsZKA: Régi-új honfoglalás és államalapítás kori bizánci pénzek a Kárpát-medencéből (Altneue byzantinische Münzen der Landnahmezeit und der ungarischen Staatsgründung aus dem Karpatenbecken). NK 102-103 (2003-2004) [2004] 149-150.

= P. ProhászKa: A vadasdi éremlelet - egy numizmatikai fikció nyomában [Der Münzfund von Vadasd - Auf der Spur einer numismatischen Fiktion]. In: A numizmatika és a társtudományok 6. Hrsg.: M. Torbágyi. Székesfehérvár 2007, 63-80.

= P. PROHÁSZKA: Bemerkungen zum byzantinischen Münzverkehr der ungarischen Landnahmezeit und der Staatsgründung im Karpatenbecken. In: Die Archäologie der frühen Ungarn. Chronologie, Technologie und Methodik. Hrsg.: B. Tobias. RGZM Tagungen 17. Mainz 2012, 67-88.

= P. PRoHÁszKa: Egy államalapítás kori bizánci soliduslelet Székelyudvarhely környékéről. Dolgozatok az Erdélyi Múzeum Érem- és Régiségtárából 6-7 (16-17) (2011-2012) [2013] 151-160.

= P. ProhászKa: Aus dem Briefwechsel von Frantisek Pošepny und Károly Torma. Im Druck.

= L. RÉVÉSZ: Erdély a magyar honfoglalás korában. Szempontok a székelyek betelepedésének kérdéséhez [Siebenbürgen zur Zeit der ungarischen Landnahme. Gesichtspunkte zur Frage der Ansiedlung der Szekler]. In: A honfoglalás kor kutatásának legújabb eredményei. Tanulmányok Kovács László 70. születésnapjára. Hrsg.: L. Révész, M. Wolf. Szeged 2013, 605-630.

= A.-M. Velter: Transilvania în secolele V-XII [Siebenbürgen im 5.-12. Jahrhundert]. Bucureşti 2002.

= V. Vollmann: Briefe zur Geschichte der siebenbürgischen Altertumskunde. Bukarest 1983.

= B. WINKLER: A verespataki aranybányászat viszonyai [Verhältnisse des Goldbergbaus von Verespatak]. FtK 1/4-5 (1871) 64-70. 